Herzschr Elektrophys 2016 $27: 73-74$ DOI 10.1007/s00399-016-0441-7

Online publiziert: 1. Juni 2016

๑) Springer-Verlag Berlin Heidelberg 2016

CrossMark

\author{
Joachim Hebe ${ }^{1}$ Sabine Ernst ${ }^{2}$ \\ 'Zentrum f. Elektrophysiologie Bremen, Am Klinikum Links der Weser, Bremen, Germany \\ ${ }^{2}$ Research Lead Electrophysiology, Royal Brompton Hospital, London, England
}

\title{
Arrhythmien bei angeborenen Herzfehlern im Erwachsenenalter
}

Betreuung in einer hierfür spezialisierten medizinischen Einrichtung. Aktuellen Schätzungen zufolge ist die Zahl an EMAH-Patienten inzwischen in Europa auf 1,8 Millionen, davon über 200.000 in Deutschland, gewachsen und übersteigt seit 2005 mit stetig zunehmendem Abstand die Zahl an AHF-Patienten im Kindes- und Jugendalter (Quelle: Nationales Register für angeborene Herzfehler e.V.).

Patienten, selbst mit milden AHF, benötigen häufig ein Leben lang medizinische Kontrollen wegen unterschiedlichster Folgen von Fehlbelastungen durch die kardialen Anomalien, selbst nach interventioneller oder chirurgischer Korrektur. Daher hat die medizinische Betreuung und Behandlung dieser $\mathrm{Pa}$ tienten zwangsläufig längst Einzug in die „Erwachsenen-Medizin/-Kardiologie" gehalten, auch wenn die absolute Zahl vergleichsweise gering ist und eine enorme Heterogenität der individuellen Ausprägungen und Zustände der kardialen Anomalien aufweist.

Neben der "Attraktivität“ des zunächst angenehm aus dem klinischen Alltag herausragenden Falles, entwickelt sich spätestens bei der Erarbeitung anstehender medizinischer Entscheidungen meist Unbehagen, da oft klinische Präsentation und mögliche therapeutische Optionen fernab der klinischen Erfahrung des Kardiologen liegen, die an erwachsenen Patienten mit „normal“ angelegtem Herz-Kreislaufsystem gesammelt wurde.

Als führendes klinisches Problem im Langzeitverlauf von Patienten mit angeborenem Herzfehler dominieren Herzrhythmusstörungen in ihrer gesamten Bandbreite Lebensqualität, Morbidität und Mortalität. Sie geben mit mehr als $50 \%$ noch häufiger Anlass für Behandlungen in ambulanter und stationärer Umgebung, als Funktionseinschränkungen des Systemventrikels und einer daraus resultierenden Herzinsuffizienz.

Die absolute Mehrheit brady- und tachykarder Herzrhythmusstörungen ist an Patienten mit normal angelegten Herzen in großer Zahl erforscht und heute meist therapeutisch beherrschbar oder gar dauerhaft zu beseitigen. Bei Patienten mit AHF treten dieselben Arrhythmieformen auf, jedoch häufig in derart verändertem Kontext und nicht selten auch in sonst ungewohnt gehäuften Kombinationen, so dass deren nichtinvasive/invasive Diagnostik sowie therapeutische Maßnahmen jeglicher Art extrem erschwert sein können. Oft sind daher Diagnostik und Therapie nur in darauf spezialisierten Händen umsetzbar.

In dieser Ausgabe von $H+E$ präsentieren wir Ihnen wesentliche Themen der Rhythmologie/Elektrophysiologie bei Erwachsenen mit AHF mit dem Ziele, den aktuellen Stand diagnostischer wie therapeutischer Möglichkeiten zusammenzufassen. Nicht zuletzt soll Ihnen eine Hilfestellung bei der gelegentlichen klinischen Mitbetreuung eines EMAHPatienten mit Herzrhythmusstörungen gegeben und aktuelle Möglichkeiten der spezialisierten Weiterversorgung aufgezeigt werden.

Kristina Wasmer aus der Arbeitsgruppe um Gerhard Diller und Lars Eckhardt, Münster, fasst die Inzidenz von Arrhythmien bei Erwachsenen mit angeborenem Herzfehler mit Blick auf die spezifischen Substrate und Arrhythmiemechanismen sowie potentielle Ursachen der Arrhythmogenese, z.B. durch chronische kardia- sich bis heute erst ein Teil der Patienten
in einer langfristigen und systematischen 
le Fehlbelastung oder Chirurgie zusammen.

Ein Überblick über Möglichkeiten des Akut- und Langzeitmanagements von brady- und tachykarden Herzrhythmusstörungen bei EMAH-Patienten wird von Gabriele Hessling, München, gegeben. Der Artikel erstreckt sich vom Notfallmanagement hämodynamisch bedeutsamer oder intolerabler Tachykardien über allgemeine Strategien kurz- oder und langfristig ausgerichteter medikamentöser und interventioneller Therapie, rhythmuschirurgischer Optionen bis hin zur potentiellen Einflussnahme durch hämodynamische Entlastung.

Das durchaus komplexe Thema der dauerhaft angelegten Geräteversorgung zur antibradykarden, antitachykarden und resynchronisierenden Therapie wird in drei aufeinanderfolgenden Artikeln behandelt. Die antibradykarde Schrittmacherversorgung, inbesondere mit Blick auf optimale Gerätewahl, Programmierung und Sondenplatzierung endowie epikardial, wird von Peter Zartner, St. Augustin zusammengefasst. Henry Chubb und Eric Rosenthal, London, UK widmen sich der Besonderheiten bei der ICD-Versorgung von EMAH-Patienten, von der Indikationsstellung bei dünnster Datenlage bis hin $\mathrm{zu}$ technischen Herausforderungen und Limitationen bei der Implantation wegen vaskulärer oder intrakardialer Unwegsamkeiten. Jan Janoussek, Prag, Tschechien, erläutert schließlich in dem Letzten der drei Artikel, ob und wie eine Resynchronisationstherapie auch bei komplexen AHF, zum Beispiel mit rechtem Systemventrikel, technisch umsetzbar ist und wann überhaupt sinnvoll.

Die invasive Elektrophysiologie kann heute auch bei Vorliegen komplexer angeborener Anomalien des Herzens oder der herznahen Gefäße erfolgreich eingesetzt werden. Die besonders im interventionellen Bereich rasant fortgeschrittene Entwicklung der Rhythmologie hat inzwischen breiten Einzug in diese Patientengruppe gehalten, da die hämodynamische Toleranz von Tachykardien oft gering und die medikamentöse Therapie nicht ausreichend verlässlich ist.
Der Stand der interventionellen Elektrophysiologie bei EMAH-Patienten ist in drei Artikel aufgeteilt, die Arbeitsgruppe von Sabine Ernst und Yen Ho, London, UK, widmet sich den atrialen Tachykardien, die sich insbesondere nach Chirurgie der AHF mit Häufigkeiten von bis zu $50 \%$ im Langzeitverlauf einstellen. Katja Zeppenfeld, Leiden, Niederlande, gibt einen Überblick über Chancen und Limitationen der Katheterablation bei ventrikulären Tachykardien. Die Interventionsmöglichkeiten der verbleibenden Gruppe mit supraventrikulären Tachykardien auf dem Boden angeborener, nicht selten auch zum AHF assoziierter Substrate wird von Joachim Hebe, Bremen zusammengefasst.

Aus der Sicht des Chirurgen stellt sich Hideki Uemura, London, UK der Frage, inwieweit heute die Errungenschaften der invasiven Elektrophysiologie bereits im chirurgischen Denken Berücksichtigung finden oder zukünftige Modifikationen chirurgischen Vorgehens bei Operationen von AHF sinnvoll erscheinen lassen.

In der Hoffnung, Ihnen mit dieser Ausgabe etwas mehr als nur eine willkommene Abwechslung zu der Flut an, zwar relevanten und zugegeben auch interessanten Hauptthemen großer Patientengruppen mit Vorhofflimmern oder koronarer Herzkrankheit zu bieten, wünschen wir Ihnen auch im Namen der Co-Autoren viel Freude, Erkenntnis und Anregung beim Lesen dieser Ausgabe,

Ihre

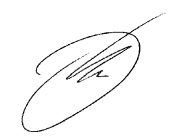

Joachim Hebe

und

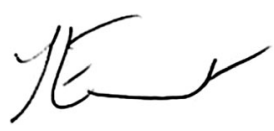

Sabine Ernst.

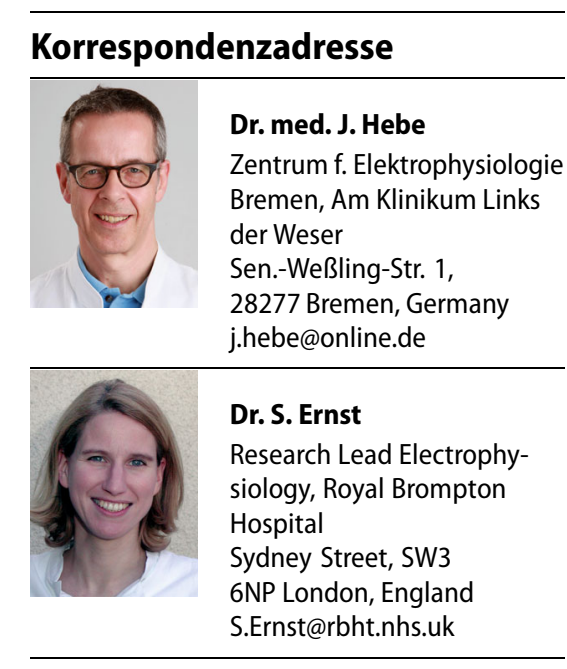

Interessenkonflikt. J. Hebe und S. Ernst geben an, dass kein Interessenkonflikt besteht. 